\title{
A Stochastic Linear Model for fMRI Activation Analyses
}

\author{
Leigh A. Johnston $^{1,2}$, Maria Gavrilescu ${ }^{3}$, and Gary F. Egan ${ }^{4,5}$ \\ 1 Electrical \& Electronic Engineering, University of Melbourne, \& NICTA Victorian \\ Research Laboratory, Australia \\ 2 Howard Florey Institute, Florey Neuroscience Institutes, Australia \\ 3 Defence Science and Technology Orginisation, Australia \\ ${ }^{4}$ Centre for Neuroscience, University of Melbourne, Australia \\ ${ }^{5}$ Monash University, Australia
}

\begin{abstract}
Purpose: The debate regarding how best to model variability of the hemodynamic response function in fMRI data has focussed on the linear vs. nonlinear nature of the optimal signal model, with few studies exploring the deterministic vs. stochastic nature of the dynamics. We propose a stochastic linear model (SLM) of the hemodynamic signal and noise dynamics to more robustly infer fMRI activation estimates. Methods: The SLM models the hemodynamic signal by an exogenous input autoregressive model driven by Gaussian state noise. Activation weights are inferred by a joint state-parameter iterative coordinate descent algorithm based on the Kalman smoother. Results: The SLM produced more accurate parameter estimates than the GLM for event-design simulated data. In application to block-design experimental visuo-motor task fMRI data, the SLM resulted in more punctate and well-defined motor cortex activation maps than the GLM, and was able to track variations in the hemodynamics, as expected from a stochastic model. Conclusions: We demonstrate in application to both simulated and experimental fMRI data that in comparison to the GLM, the SLM produces more flexible, consistent and enhanced fMRI activation estimates.
\end{abstract}

Keywords: fMRI, hemodynamic signal, Kalman filter, state space model.

\section{Introduction}

The mapping from neuronal activation to measured blood oxygenation level dependent (BOLD) signal in fMRI involves a complex interplay between physiological and physical processes, most of which are yet to be fully understood [7]. The hemodynamic response function (HRF) is known to vary across subjects, sessions, scans, and brain regions [6]. Approaches to dealing with this variability in HRF have, with few exceptions, focussed on parameterised, deterministic models of the HRF, eg. 14]. Parameterised models may appear at first glance to be stochastic, given that estimated parameters are assumed to be drawn from prior distributions. However, given knowledge of the parameters, the resultant HRFs are deterministic. A notable exception to the deterministic component 
models are the techniques originated by Ciuciu et al. [3], in which smooth HRFs are ensured by placing a Gaussian prior on the norm of the second derivative of the HRF, with estimates enforced to start and end at zero.

In contrast to deterministic BOLD signal models, we propose a stochastic linear model (SLM) of the BOLD signal, in which noise is used to drive the state dynamics directly. Unlike the methods of Ciuciu et al., our model is stochastic and parameterised, with the BOLD signal estimated voxelwise along with the activation weight. The SLM is a state space model, similar in form to the bilinear dynamical systems (BDS) proposed by [12] and recently extended by [10]. The key difference between the SLM and BDS is that BDS models the supposed neuronal activity via stochastic state dynamics, which are then convolved with HRF kernels to form the BOLD signal. Given the vast difference in temporal scale between fMRI observations and neuronal activity, the SLM does not attempt to separate neuronal activity from the hemodynamic response. Rather, the SLM is a fully stochastic model of the hemodynamic BOLD signal.

The primary focus of the current paper is to present the SLM as a stochastic variant of the general linear model (GLM), and to compare the robustness, statistical significance and consistency of activation estimates derived from the SLM and GLM approaches, rather than to explicitly estimate a correlate of neuronal activity.

\section{Methods}

The Stochastic Linear Model: Let $k=1, \ldots, T$ be the discrete time index of repetition time (TR) intervals. The system input is a binary stimulus sequence, $u_{k}$, representing task-on/task-off, and system output is the observed voxelwise BOLD signal, $y_{k}$. The SLM is a state space model consisting of an ARX state equation modelling the hemodynamic signal, $x_{k}$, and an observation equation:

$$
\begin{aligned}
& x_{k}=a x_{k-1}+b u_{k}+w_{k}, \quad w_{k} \sim N\left(0, \sigma_{w}^{2}\right), \\
& y_{k}=x_{k}+v_{k}+r_{k}+e_{k}, \quad v_{k} \sim A R\left(c, \sigma_{n}^{2}\right), r_{k} \sim M A\left(d, \sigma_{\xi}^{2}\right), e_{k} \sim N\left(0, \sigma_{e}^{2}\right) .
\end{aligned}
$$

For full generality, we consider additive autoregressive (AR), moving average (MA) and Gaussian white noise processes in the observation equation.

The SLM can be represented in canonical state space form by embedding the ARMA noise processes in the state vector. For the MA noise process, state embedding occurs via defining a dummy variable, $\tilde{r}_{k}=d \xi_{k}$. Let $\mathbf{x}_{k}=\left[x_{k}, v_{k}, r_{k}, \tilde{r}_{k}\right]^{\prime}$ be the state vector and $\mathbf{w}_{k}=\left[w_{k}, n_{k}, \xi_{k}, d \xi_{k}\right]^{\prime}$ be the noise vector. Then the SLM canonical state space form is,

$$
\begin{aligned}
& \mathbf{x}_{k}=A \mathbf{x}_{k-1}+\mathbf{b} u_{k}+\mathbf{w}_{k}, \quad \mathbf{w}_{k} \sim N(0, Q), \\
& y_{k}=\mathbf{g}^{\prime} \mathbf{x}_{k}+e_{k}, \\
& A=\left[\begin{array}{llll}
a & 0 & 0 & 0 \\
0 & c & 0 & 0 \\
0 & 0 & 0 & 1 \\
0 & 0 & 0 & 0
\end{array}\right], \quad \mathbf{b}=\left[\begin{array}{l}
b \\
0 \\
0 \\
0
\end{array}\right], \quad Q=\left[\begin{array}{cccc}
\sigma_{w}^{2} & 0 & 0 & 0 \\
0 & \sigma_{n}^{2} & 0 & 0 \\
0 & 0 & \sigma_{\xi}^{2} & d \sigma_{\xi}^{2} \\
0 & 0 & d \sigma_{\xi}^{2} & d^{2} \\
\sigma_{\xi}^{2}
\end{array}\right], \quad \mathbf{g}=\left[\begin{array}{l}
1 \\
1 \\
1 \\
0
\end{array}\right] .
\end{aligned}
$$


The full state vector, $\mathbf{x}_{k}$, and the ARMA noise processes, $v_{k}$ and $r_{k}$, are estimated from the noisy BOLD signal, $y_{k}$.

There are three key differences between the SLM and the GLM. Firstly, the SLM dynamics of the neurovascular signal, $x_{k}$, are driven by a state noise process, $w_{k}$, whereas the GLM neurovascular signal dynamics are deterministic. Secondly, unlike the GLM, the SLM does not contain an explicit activation parameter, $\beta$, or hemodynamic response function, $h_{k}$. This permits a more flexible modelling of the neurovascular signal where the HRF may be unknown. In order to map the strength of activation, we define an SLM activation weight following in Eq. (4). The third key difference is that in the GLM, attempts are made to mitigate the effect of observation noise through pre-whitening or pre-colouring of the measured BOLD signals [4. In contrast to these noise suppression procedures, the SLM noise processes are explicitly estimated, through embedding them in the state dynamics.

SLM Activation Estimation: While the activation weight, $\beta^{G L M}$, is explicit in a GLM formulation, there is no analogous parameter in the SLM state or observation equation, Eq. (1). Rather we define the activation weight to be

$$
\beta^{S L M} \triangleq \frac{b}{1-a}
$$

where $a$ and $b$ are SLM parameters in Eq. (1). This definition is derived directly from the SLM state equation, Eq. (1), by noting that $x_{k}$ contains stimulus terms in an arithmetic sequence due to the autoregression. We therefore define the activation weight to be the limiting sum of the arithmetic sequence, Eq. (4). Activation weights for higher order AR models can be defined similarly using the characteristic polynomial in the denominator of (4).

State and parameter estimation: The proposed SLM algorithm estimates the noise processes explicitly, rather than suppressing their effect through prewhitening. The SLM parameters $\Theta=\left\{a, b, c, d, \sigma_{e}^{2}, \sigma_{w}^{2}, \sigma_{n}^{2}, \sigma_{\xi}^{2}\right\}$ are unknown, as are the state vectors, $\mathbf{x}_{k}$. Given that both states and parameters are unknown, we seek to estimate the MAP estimates of the states and parameters,

$$
\{X, \Theta\}^{M A P}=\arg \max _{X, \Theta} p\left(X, \Theta \mid y_{1}, \ldots, y_{T}\right)
$$

where $\quad p\left(X, \Theta \mid y_{1}, \ldots, y_{T}\right)=\prod_{k=1}^{T} \frac{1}{\sqrt{2 \pi \sigma_{e}^{2}}} \exp \left(-\frac{\left(y_{k}-\mathbf{g}^{\prime} \mathbf{x}_{k}\right)^{2}}{2 \sigma_{e}^{2}}\right) \times$

$$
\frac{1}{(2 \pi)^{2}|Q|^{\frac{1}{2}}} \exp \left(-\frac{1}{2}\left(\mathbf{x}_{k}-A \mathbf{x}_{k-1}-\mathbf{b} u_{k}\right)^{\prime} Q^{-1}\left(\mathbf{x}_{k}-A \mathbf{x}_{k-1}-\mathbf{b} u_{k}\right)\right)
$$

This MAP optimisation is intractable, and therefore a suboptimal estimation algorithm must be applied. We have chosen iterative coordinate descent algorithm [9], known to converge to a local minimum of the posterior, in which a Kalman smoother and parameter updating are consecutively applied to the system, starting from initial parameter estimates. The computational time of 
the iterative estimation is less than 1 second per voxel time-series (for a scan length of 240 volumes), implemented in Matlab and run on a standard $2 \mathrm{GHz}$ $\mathrm{PC}$. The algorithm is entirely parallelisable as each voxel analysis is independent of others.

Simulated fMRI Data: Event design data was generated by the balloon model, through random sampling over a realistic range of parameters [5]. SNR was varied by change in neuronal efficacy, $\varepsilon$. Parameters were generated in each of 50 runs according to

$$
\begin{aligned}
& T=150 s, T R=2 s, a_{1}=1, a_{2}=3.37, \alpha \sim N(0.33,0.1), E_{0} 0.3+0.3 U(0,1), \\
& \tau_{s} \sim N(1.54,0.2), \tau_{f} \sim N(2.46,0.2), \tau_{0} \sim N(1,0.2), V_{0} / \sigma_{e}^{2}=5
\end{aligned}
$$

An estimated activation weight was computed for each run based on the peak of the noiseless BOLD signal, for comparison with $\beta_{G} L M$ and $\beta_{S} L M$. Mean square error between the noiseless BOLD signal and the estimated signal from the GLM and SLM methods was similarly computed.

Experimental fMRI Visuomotor Task Data: Three healthy controls were scanned (3T Siemens TRIO, MCRI Melbourne) while performing a visuomotor task, 222 EPI images, in-plane: $3.125 \times 3.125 \mathrm{~mm}^{2}, \mathrm{TR}=1.6 \mathrm{~s}, \mathrm{FA}=90^{\circ}, \mathrm{TE}=20 \mathrm{~ms}$, 24 axial slices of $5 \mathrm{~mm}$ with $0.5 \mathrm{~mm}$ gap. The visuomotor task was a simple block design of alternating periods (30s each). Images were motion corrected and spatially smoothed with a $6 \mathrm{~mm}$ isotropic Gaussian kernel. The supplementary motor area (SMA) was expertly delineated using landmarks in each subject's native space. The SLM's iterative coordinate descent algorithm was applied for 20 iterations, determined to be sufficient to allow the parameter estimates to converge. For comparison, a GLM using the SPM canonical HRF and temporal derivatives was implemented.

\section{Results}

Simulations: Simulated data that has been generated using the Balloon model, rather than the actual models underlying SLM or GLM, avoids the problem of bias toward a particular algorithm in the results. The Balloon model, being based on physiology, is also a more realistic model from which to generate data. Varying the 'efficacy' parameter, $\varepsilon$, in the Balloon model [5], varies the cerebral blood flow and hence BOLD signal. Fig. 11 demonstrates that for the simulated event design data, the SLM achieves more accurate estimates of activation weights than the GLM, which consistently underestimates the signal level. The SLM returned a lower MSE than the GLM for almost all efficacies (SNRs), excepting the extremely low SNR regime; as expected the SLM will tend to overfit the signal and match to noise in very low SNR BOLD signals that are dominated by noise. 

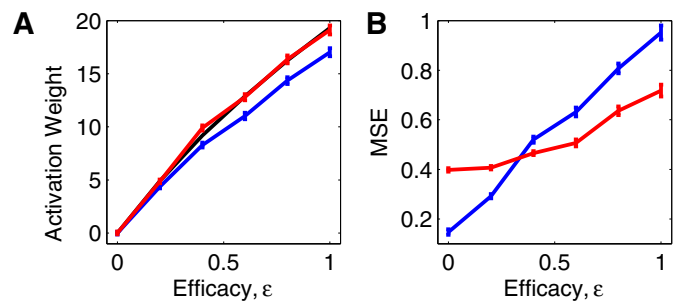

Fig. 1. Performance comparison between SLM and GLM on simulated event design data, simulated for six signal efficacies, $\varepsilon \in[0,1]$. A. Ground-truth activation weights (black) vs SLM (red) and GLM (blue). Where not visible, black line underlies SLM red line. B. MSE between ground-truth time-series and each of SLM (red) and GLM (blue) estimates. All estimates display mean \pm standard error bars over 50 runs.

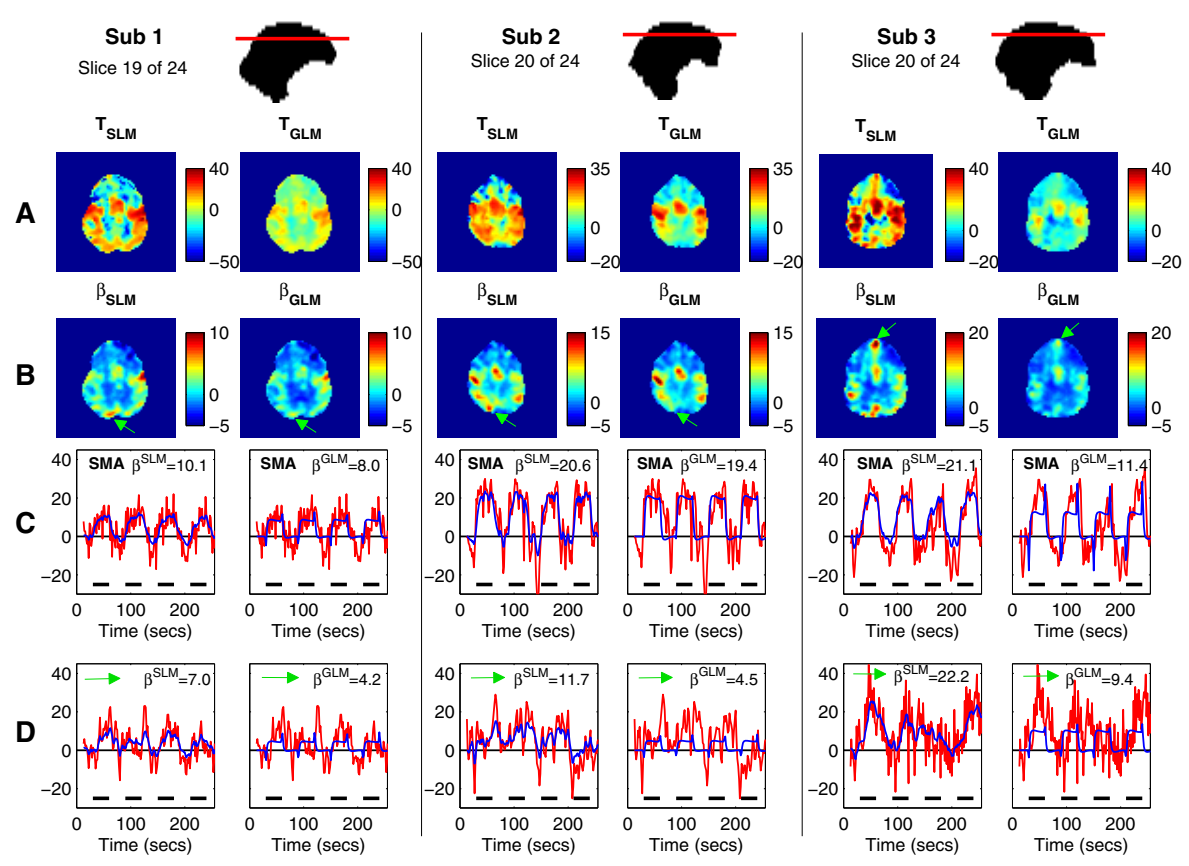

Fig. 2. Estimation of BOLD signal dynamics by SLM and GLM across 3 subjects for a visuomotor block design task. A. T statistics. B. Activation estimates. C. SMA timeseries (red) and estimates (blue). D. Time-series (red and estimates (blue) for voxels labelled by green arrows in $\mathbf{B}$. 
Experimental Results: Fig. 2/A and B depict the T statistic and estimated activation maps for a representative slice in each subject containing the motor regions as identified manually by an expert. T statistics for the SLM were computed as detailed in App. A. The T statistic maps display a greater range of values with particularly stronger activation in expected motor areas. The SLM activation maps tend to be more punctate than those of the GLM. Fig. $2 \mathrm{C}$ displays the observed BOLD signal in the SMA and the corresponding SLM and GLM estimates. For comparison purposes, the same display is presented in Fig. 2D for a second voxel chosen in each subject at a region determined to be activated more strongly by the SLM than the GLM method. These voxels are indicated by green arrows in Fig. 2B. Comparison of the time-series estimates demonstrate that the SLM is more consistent in assignment of activation weights than the GLM, in the following ways: In Subject 1 SMA, the SLM and GLM activation weights are similar (10.1 and 8.0 respectively as indicated on the figures). The GLM fails to capture the shape of the comparison voxel's time-series in Fig. 2D however, resulting in a halving of $\beta^{G L M}$, while the SLM determines a reasonable activated time-series shape. The same comment applies to Subjects 2 and 3; The SLM activation weight between SMA and the comparison voxel are reasonable given the relative time-series shapes, while the GLM fails to be able to account for any activation in the comparison voxels. Indeed, in Subject 3, the GLM estimate in the SMA is a poor fit to the data that results in a halved activation weight $\left(\beta^{G L M}=11.4\right)$ compared to the SLM SMA $\left(\beta^{S L M}=21.1\right)$.

\section{Discussion and Conclusion}

The use of a state noise to drive the system dynamics is fundamentally different to the inclusion of AR noise in the observation equation, as in 141311. The model proposed by [1] is similar to the SLM, however the ARX model is presented as an observation equation and is thus reduces to a deterministic model in high SNR.

The SLM is implicitly Bayesian, in that the state equation is equivalently a prior on the signal dynamics, and the resultant estimation algorithm optimises in a maximum a posteriori sense for the best state and parameter estimates given the observed data. Our approach differs to Bayesian models in the literature, for example [1410, in that we have not placed prior distributions governed by hyperparameters on the signal and noise parameters. Such prior distributions can be added at the cost of computational expense. The purpose of the current model is to provide an alternative to the GLM without the use of prior distributions on the parameters.

The stochastic nature of our hemodynamic signal model results in the ability to reshape the input sequence, $u_{k}$, to better fit the observed BOLD signal in each voxel. Overfitting of data by models with many parameters is always a 
concern. Our simulations and experimental results have demonstrated that the SLM refines the GLM estimates, suggesting that the SLM parameterisation well represents the state and observation processes rather than making overfit inferences. What has previously been described as the nonlinearity of the HRF [2] is now placed in the domain of deterministic vs stochastic. It is of interest to compare in future the SLM with methods in which parameterised, deterministic HRFs are estimated [14], or those in which the HRF is constrained to be a smoothly varying function [3]. Similarly, in future work we will compare the stochastic linear model with nonlinear extended balloon models of the BOLD signal [8] and their estimation strategies, and alternative stochastic modelling approaches such as BDS [1012.

We have established the stochastic linear model as an alternative to the general linear model for mapping activation, hemodynamic dynamics and noise processes in BOLD fMRI experiments. The SLM provides an inherently flexible model that makes use of uncertainty to drive the estimated hemodynamic signal dynamics, thus accounting for non-canonical responses. Unlike other stochastic models of the BOLD signal, the SLM does not attempt to separate out neuronal activity from the hemodynamics, a decision reflecting the lack of available ground truth at the level of neuronal activity for fMRI experiments.

\section{A SLM Significance Testing}

The SLM activation weights are calculated according to Eq. (4), from parameter estimates of $a$ and $b$ at the $M^{\text {th }}$ iteration, $a^{(M)}$ and $b^{(M)}$, which are themselves the result of a least squares optimisation of the objective function. Therefore the variances of the parameter estimates are known.

T-statistics for $a^{(M)}$ and $b^{(M)}$ are formed by dividing the estimates by their respective standard deviations. The activation estimate, $\beta^{S L M}$, is a ratio between estimates $b^{(M)}$ and $\left(1-a^{(M)}\right)$. While it is known that the ratio of two Gaussian random variables produces a Cauchy-distributed random variable, the distribution of the ratio of non-zero-mean T-distributed estimates is considerably more involved. Through simulations, we have empirically derived the following expression for the standard deviation of $\beta^{S L M}$,

$$
\operatorname{std}\left(\beta^{S L M}\right)=\operatorname{std}\left(b^{(M)}\right)\left(1-a^{(M)}\right),
$$

that is, interestingly, independent of standard deviation of $a^{(M)}$. This expression enables transformation of $\beta^{S L M}$ to a t-statistic as per $a^{(M)}$ and $b^{(M)}$ above.

To validate the empirical relationship in Eq. (7), we present the results of simulations of the SLM state equation, Eq. (11), for a range of $a$ and $\sigma_{w}^{2}$. Changes in $b$ modulate the mean without affecting variances, therefore this is chosen to be $b=1$ without loss of generality. The stimulus $u_{k}$ is from the experimental visuomotor data, thus $T=215.5000$ runs were simulated per parameter pair. 


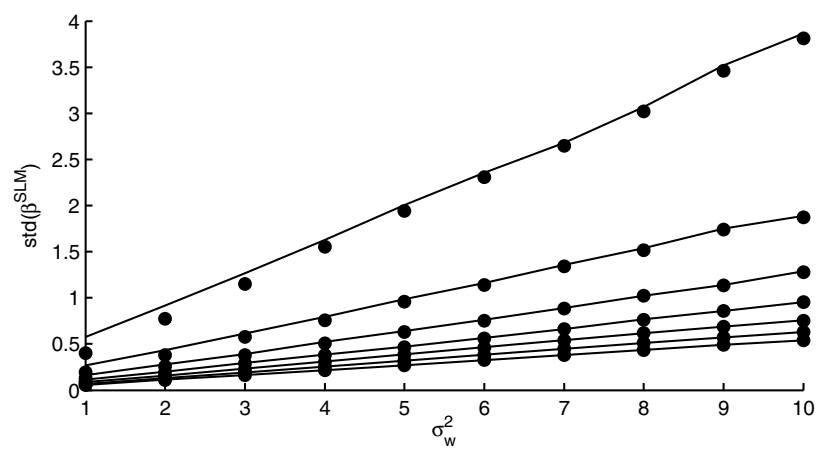

Fig. 3. Simulations validating the empirical relationship in Eq. (7). Standard deviation of $\beta^{S L M}$ calculated at each parameter $a \in\{-0.75,-0.5,-0.25, \ldots, 0.75\}$ (data sets bottom to top of plot) from 5000 simulation runs (dots) and via Eq. (7) (solid lines).

Fig. 3 depicts the standard deviation of the $\beta^{S L M}$ estimates along with the empirical relationship in Eq. (7) for each parameter pair, in close agreement. Skewness and kurtosis of the $\beta^{S L M}$ estimates were concordant with a Gaussian.

\section{References}

1. Baraldi, P., Manginelli, A.A., Maieron, M., Liberati, D., Porro, C.A.: An ARX model-based approach to trial by trial identification of fMRI-BOLD responses. NeuroImage 37, 189-201 (2007)

2. Birn, R.M., Saad, Z.S., Bandettini, P.A.: Spatial heterogeneity of the nonlinear dynamics in the FMRI BOLD response. NeuroImage 14, 817-826 (2001)

3. Ciuciu, P., Poline, J.B., Marrelec, G., Idier, J., Pallier, C., Benali, H.: Unsupervised robust nonparametric estimation of the hemodynamic response function for any fMRI experiment. IEEE TMI 22(10), 1235-1251 (2003)

4. Friston, K.J., Josephs, O., Zahahn, E., Holmes, A.P., Rouquette, S., Poline, J.B.: To smooth or not to smooth? Bias and efficiency in fMRI time-series analysis. NeuroImage 12, 196-208 (2000)

5. Friston, K.J., Mechelli, A., Turner, R., Price, C.J.: Nonlinear responses in fMRI: The Balloon model, Volterra kernels, and other hemodynamics. NeuroImage 12, 466-477 (2000)

6. Handwerker, D.A., Ollinger, J.M., D'Esposito, M.: Variation of BOLD hemodynamic responses across subjects and brain regions and their effects on statistical analyses. NeuroImage 21, 1639-1651 (2004)

7. Heeger, D.J., Ress, D.: What does fMRI tell us about neuronal activity? Nature Reviews Neuroscience 3, 142-151 (2002)

8. Johnston, L.A., Duff, E.P., Mareels, I., Egan, G.F.: Nonlinear estimation of the BOLD signal. NeuroImage 40, 504-514 (2008)

9. Luenberger, D.G.: Linear and Nonlinear Programming, 2nd edn. Addison Wesley, Reading (1984) 
10. Makni, S., Beckmann, C., Smith, S., Woolrich, M.: Bayesian deconvolution fMRI data using bilinear dynamical systems. NeuroImage 42, 1381-1396 (2008)

11. Makni, S., Ciuciu, P., Idier, J., Poline, J.: Joint detection-estimation of brain activity in fMRI using an autoregressive noise model. In: IEEE ISBI, pp. 1048-1051 (2006)

12. Penny, W., Ghahramani, Z., Friston, K.J.: Bilinear dynamical systems. Phil. Trans. R. Soc. B 360(1457), 983-993 (2005)

13. Purdon, P.L., Solo, V., Weisskoff, R.M., Brown, E.N.: Locally regularized spatiotemporal modeling and model comparison for functional MRI. NeuroImage 14, 912-923 (2001)

14. Woolrich, M.W., Jenkinson, M., Brady, J.M., Smith, S.M.: Fully Bayesian spatiotemporal modeling of FMRI data. IEEE TMI 23(2), 213-231 (2004) 